\title{
Kapitel 2. \\ Der Auftakt - Dionysos und die Satyrn
}

\author{
For as long as this vaulted collusion lasts, \\ a mood of swooning Dionysiac laxity roves the air.
}

Patrick Leigh Fermor ${ }^{1}$

\subsection{Der Kontext}

Wesentliche Anhaltspunkte zum Verständnis der Ampelos-Episode in all ihren Detailaspekten und in ihrer kompositorischen Gesamtheit und Relation zum EposGanzen liefert bereits die Eröffnungsszene (Nonn. D. 10,139-174), in welcher der erzählerische Bogen gespannt und Schauplatz sowie Hauptfiguren vorgestellt werden. Diese Scharnierstelle im Epos erlaubt es, Rückschlüsse auf größere kompositorische Zusammenhänge zu ziehen: Im 10. Buch der Dionysiaka bringt Nonnos die Erzählungen um die Kindheit des Dionysos und das Los seiner Familie zu einem Abschluss, nachdem er zuvor in aller Ausführlichkeit die mythischen Wurzeln des Weingottes bis zu dessen erstem Auftritt behandelt hat. Die Antizipation wichtiger Motive und Leitgedanken, die für den weiteren Verlauf der Erzählung um Dionysos Relevanz zeigen und die die Ampelos-Episode sowie ihr narratives Ziel, die Genese des Weines, vorbereiten, sind die wesentlichen Aspekte nonnianischer Dichtungsprinzipien.

In der Schilderung der mythischen Urgeschichte und ihrer handelnden Figuren werden Eigenschaften des späteren Weingottes sowie sein dionysischer Charakterzug geprägt und vorgezeichnet, indem etwa schon Kadmos, Dionysos' Großvater, in den ersten fünf Büchern im Verein mit Zeus gegen den Usurpator Typhon kämpft (Nonn. $D$. 1 und 2), auf der Suche nach seiner Schwester Europa in die Welt hinauszieht, nach Griechenland gelangt, Theben gründet und so das spätere Wirken des Dionysos vorbereitet (Nonn. D. 3 und 4). Durch Kadmos werden zivilisatorische und kulturtechnische Fähigkeiten ebenso vorweggenommen wie durch dessen Kinder Polydoros, Autonoe, Ino, Agaue und Semele (Nonn. D. 5). Anstatt eine durchgehende Erzählung über das Schicksal der Familie des Kadmos zu bieten, unterbricht der Erzähler an der Stelle, an der Semele erstmals genannt wird: Ihre Bestimmung wird lediglich angedeutet, die Verbindung mit Zeus und die Geburt ihres gemeinsamen Sohnes Dionysos auf einen späteren Punkt innerhalb der Erzählung verschoben. ${ }^{2}$ Im 6. Buch werden

1 Fermor (2004) 163.

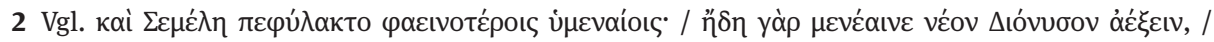

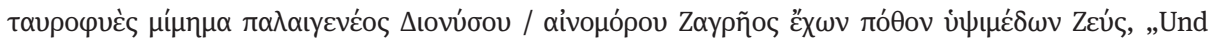
Semele war aufgespart für eine noch glänzendere Hochzeit: Denn er wollte einen neuen Dionysos heranreifen lassen, ein stiergestaltiges Abbild des einstmals geborenen Dionysos, des Zagreus, nach 
Zeus’ Liebesabenteuer mit Persephone, die Geburt, das kurze Leben und der Tod des Zagreus eingeschaltet. Insbesondere diese Episode nimmt Aspekte des Weingottes vorweg, die in den aufeinanderfolgenden Göttergenerationen wiederkehren werden: Der illegitime Zeus-Sohn soll in die Fußstapfen seines Vaters treten, ihn ereilt jedoch durch die Hand der Titanen ein vorzeitiges Ende, Weltenbrand und Sintflut beenden die mythische Urzeit, die in der mythischen Chronologie vor dem Erscheinen des Weingottes anzusetzen ist.

Die Bücher 7-9 schildern die Ereignisse um Dionysos' doppelte Geburt und Erziehung. Mit der anschließenden Erzählung um Ino und Athamas wird die Familiengeschichte wieder aufgenommen, gleichzeitig aber eine weitere Komponente des Dionysischen eingeführt, der Wahnsinn der Ino und des Athamas, der als Ausprägung dionysischen Wahnsinns an mehreren Stellen in den Dionysiaka eine Rolle spielen wird (Nonn. D. 9,243-321; 10,1-138). Die narrative Technik der Vorwegnahme, des InBeziehung-Setzens zweier oder mehrerer Szenen, die weder in einem ursächlichen Zusammenhang miteinander stehen noch sich in eine klar definierte chronologische Abfolge bringen lassen, ist symptomatisch für Nonnos' dichterisches Programm und essentiell für das Verständnis der Ampelos-Episode. Es geht dem Dichter nicht darum, ein Epos zu schaffen, das durch kausale Handlungsstringenz zusammengehalten wird, sondern vielmehr um die Reprise von Motiven und Leitthemen, die für den Protagonisten Dionysos Relevanz zeigen. Trotz des unerwartet raschen Schwenks von der InoAthamas- zur Ampelos-Episode werden beide durch den einenden Faktor Wasser aneinander gebunden, welcher für beide Szenen konstitutiv ist.

\subsection{Protagonist und Schauplatz}

Auf Inos und Melikertes' Sprung ins Meer (Nonn. D. 10,120-125) und die Schmährede Semeles gegen Ino (10,126-138) folgt abrupt und übergangslos die Ampelos-Episode. Der Dichter lenkt mitten im 10. Buch den erzählerischen Fokus auf einen völlig anderen Schauplatz (Nonn. D. 10,139-141): ${ }^{3}$

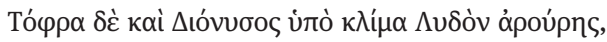

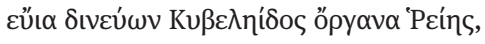
140

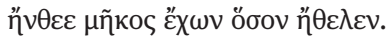

Und während Dionysos im Gebiet des lydischen Landes (140) die bakchischen Instrumente zu Ehren der kybelischen Rheia schwang, kam er in die Blüte seiner Jahre und hatte die Körpergröße erreicht, wie er sie wollte.

dem der hochwaltende Zeus Sehnsucht hatte, der aber ein schreckliches Ende fand“ (Nonn. D. 5,562565).

3 Zur narrativen Funktion der Eröffnungsszene der Ampelos-Episode siehe auch Gigli Piccardi (2003) 628-633; Kröll (2013b). 
Der junge Dionysos wird in den ersten zweieinhalb Versen als Protagonist der folgenden Erzählung vorgeführt und in Lydien, seinem Heimatland, verortet, das für die kommenden zweieinhalb Bücher den Schauplatz der Handlung bilden wird. Bereits in den ersten drei Versen der Ampelos-Episode erfolgt die Präsentation von Hauptfigur und Ort, wobei Dionysos bei seinem erstmaligen Auftritt als selbstständig agierende Figur in seiner Rolle als Gott sichtbar wird: Er schwingt seine göttlichen Attribute (عủı $\alpha$ [...] őpyava [10,140]) und verfügt über herausragende göttliche Macht, die es ihm ermöglicht, die vollständige Kontrolle über seine menschliche Erscheinungsform aus-

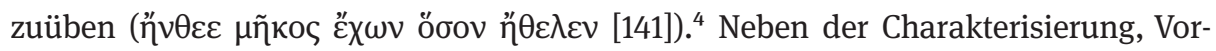
stellung und Einordnung einer der Hauptfiguren der Ampelos-Episode nimmt der Dichter zur Problematik der Göttlichkeit des Dionysos Stellung, die seit der Geburt des außerehelichen Zeus-Sohnes immer wieder thematisiert wird, und lässt Dionysos bewusst zwischen den Extremen Gott und Mensch changieren.

Mit der Darstellung des Schauplatzes - des Flusses Paktolos - verfolgt der Dichter eine narrative Strategie und liefert eine ausführliche, in mehreren Einstellungen durchgeführte Ekphrasis des Ortes, an dem sich Dionysos gerade befindet, sowie der Tageszeit, zu der sich die Geschehnisse abspielen (Nonn. D. 10,141-147):

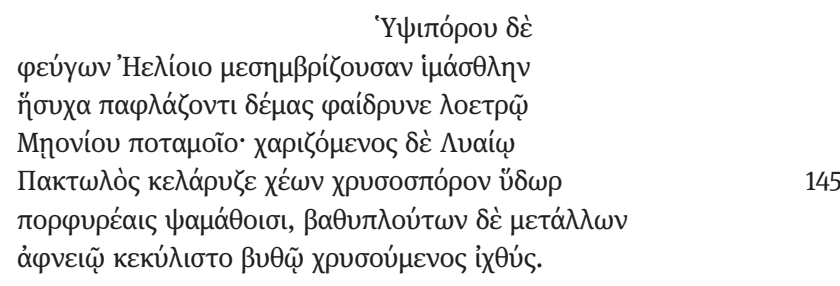

Um vor der mittäglichen Geißel des auf luftigen Pfaden wandelnden Helios zu fliehen, machte er seinen Leib strahlend durch ein ruhig plätscherndes Bad im maionischen Fluss. Um sich Lyaios freundlich zu zeigen, (145) ließ es Paktolos gurgeln und schüttete sein goldsamiges Wasser auf die purpurnen Sande aus. Und auf dem von reichen Metallen in der Tiefe überbordenden Grund zappelte der güldene Fisch.

Der Blick des Lesers, der zunächst gleichsam aus der Vogelperspektive von einem erhöhten Standpunkt auf ganz Maionien fällt, ${ }^{5}$ wird innerhalb weniger Verse auf ein konkretes Flussufer fokussiert, ja noch weiter gebündelt und bis auf den Grund des Paktolos hinabgeführt. Die detaillierte Beschreibung des Ortes nimmt dessen herausragende Bedeutung für die bevorstehende Handlung vorweg, indem das rhetorische Genus der Ekphrasis gezielt im Hinblick auf das kompositionelle Vorhaben

4 Vgl. Call. Hec. 277 Pfeiffer (= 279 Asper) mit einer klanglichen Assoziation an die Stelle bei Nonnos:

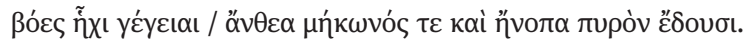

5 Bei Maionien handelt es sich um einen anderen Namen für Lydien, vgl. Chuvin (1991) 99f.; zur besonderen Rolle Lydiens in den Dionysiaka siehe Chuvin (2009) 187. 
eingesetzt wird: ${ }^{6}$ Beinahe die gesamte Ampelos-Episode spielt am Paktolos, der Hauptschauplatz Flussufer wird nur zweimal - vorübergehend - verlassen. ${ }^{7}$ Die in Buch 12 erfolgende Geburt des Weinstocks durch die Metamorphose des Ampelos ereignet sich in ebendieser Umgebung, der dem Dionysos heilige Fluss schafft somit die Rahmenbedingungen für den Höhepunkt der Episode. Das Paktolos-Ufer wird außerdem zum idealen Ort für die Liebesgeschichte zwischen Dionysos und Ampelos, da die durch Blumen und üppige Vegetation gekennzeichnete Landschaft in der Tradition idyllisch-erotischer Szenen mit aitiologischem Gehalt steht, wie sie etwa für die hellenistische Dichtung typisch sind. Die Schönheit der Szenerie präfiguriert die Schönheit des Ampelos und die Vollkommenheit der Liebe des Dionysos zu seinem Begleiter. ${ }^{8}$

Mit der Wahl des Paktolos als Bühne des Geschehens rührt Nonnos an einen mythisch-literarischen Gemeinplatz, der den Paktolos bereits in der klassischen Antike als den Gold führenden Fluss schlechthin stilisiert, so etwa bei Herodot und Strabo. ${ }^{9}$ Nonnos betont ebenfalls den Goldreichtum des Flusses, ordnet ihn in das narrative Programm seines Epos ein und hebt insbesondere seine dionysische Komponente hervor. ${ }^{10}$ Zusammen mit einigen weiteren Toponymen der Region Lydien taucht der Fluss im Abschnitt über die Lyder auf, die sich mit einem Heereskontingent an Dionysos' Zug gegen die Inder beteiligen (Nonn. D. 13,464-473); ein Teil der lydischen Streitkräfte stammt aus diesem Gebiet, ${ }^{11}$ wieder andere aus Kerassa, einer Stadt, die ihren Bezug zu Dionysos bereits im Namen trägt: An diesem Ort mischte Dionysos erstmals Wasser mit Wein. ${ }^{12}$ Indem der als locus amoenus präsentierte Paktolos den

6 Zur Ekphrasis bei Nonnos vgl. Accorinti (2009) 93-95. Deskription bildet ein wesentliches kompositorisches Element der Ampelos-Episode; zur Funktion der Ekphrasis in den Büchern 10-12 der Dionysiaka siehe Kap. 6.5.

7 Als weitere Teilschauplätze dienen das Gebirge und der Palast des Helios: Der Satyr Ampelos entfernt sich vom Fluss und wird im Gebirge getötet; mit dem Besuch der Personifikationen der vier Jahreszeiten bei Helios erfolgt ein Schwenk auf die Götterebene. Zu Raum und Schauplatz der Ampelos-Episode siehe S. 201-205.

8 Zur erotischen Konnotation von Naturbildern in der hellenistischen Literatur vgl. Calame (1999) 153-164.

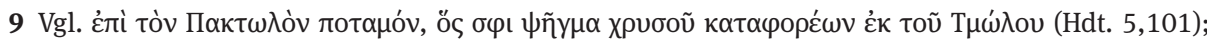

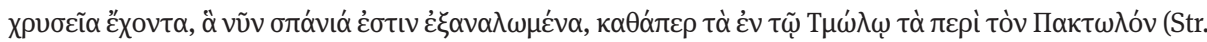

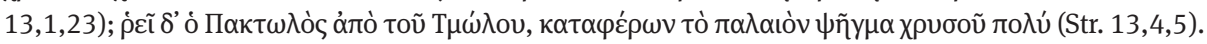
10 Zum Paktolos als Symbol Lydiens in den Dionysiaka vgl. Nonn. D. 33,258 und 47,27; zum Goldreichtum des Flusses siehe Nonn. D. 11,312; 12,127; 13,472; 22,148; 25,332; 37,115 und 129; 41,86; 43,411 und 442; 47,599; vgl. auch Chrétien (1985) 138f. Anm. ad 145; Vian (1995) 156.

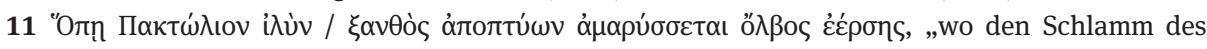
Paktolos der goldgelbe Reichtum des Wassers funkelnd von sich gibt“ (Nonn. D. 13,472f.).

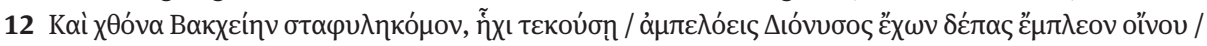

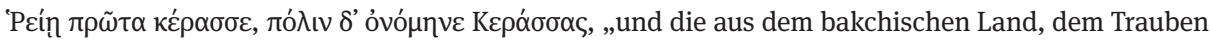
hegenden, wo für seine Mutter der rebenreiche Dionysos einen Becher in der Hand hielt und mit Wein füllte und für Rheia zum ersten Mal [mit Wasser] mischte; und die Stadt nannte er Kerassa“ (Nonn. D. 13,468-470), vgl. Chuvin (1991) $104 f$. 
Bezugsrahmen für die unmittelbar bevorstehenden Ereignisse um die Entstehung des Weines und die Ausstattung des Gottes mit ebendiesem Attribut bildet, ${ }^{13}$ gehen der Protagonist Dionysos und der Schauplatz, das Paktolos-Ufer, eine enge Verbindung miteinander ein. Das Programm der Ampelos-Episode ist von Anfang an abgesteckt: Am Ende der Erzählungen um Dionysos und Ampelos steht die Etablierung und Anerkennung des Weingottes.

\subsection{Die Figuren}

Der lydische Paktolos, der an dieser Stelle erstmals in den Dionysiaka Erwähnung findet, ist nicht bloße Kulisse für die bevorstehende Handlung, sondern huldigt durch sein Auftreten dem Weingott Dionysos und spielt einen aktiven Part in der Ein-

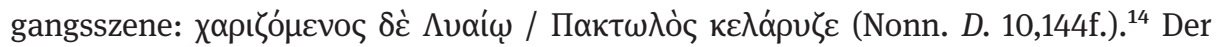
Eindruck von Dynamik wird zusätzlich durch den im Fluss badenden Dionysos sowie die im Wasser zappelnden Fische gesteigert, Nonnos' Freude an der Schilderung von Bewegung gipfelt schließlich in den ebenfalls im Paktolos spielenden Satyrn (Nonn. $D$. 10,148-168):

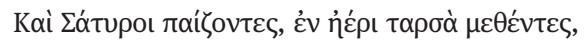

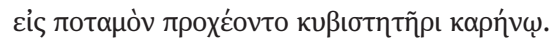

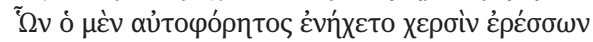
150

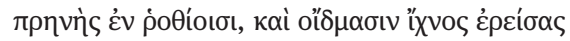

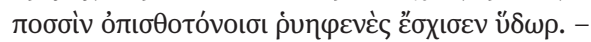

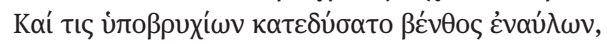

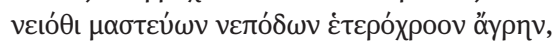



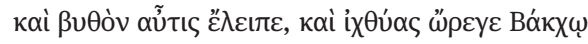

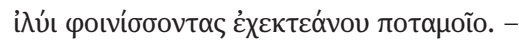

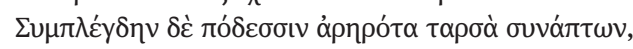

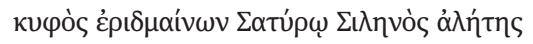

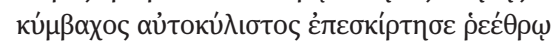

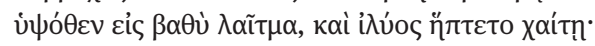

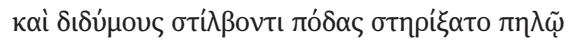

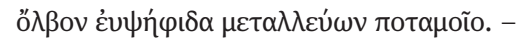

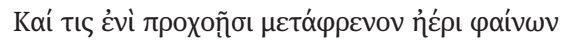

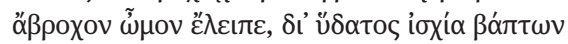

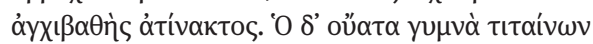

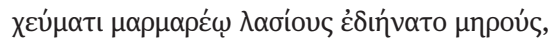

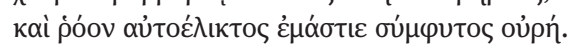

13 Ein locus amoenus bildet auch den narrativen Rahmen für die Brotvermehrung Jesu in Nonn. P. 6,152, vgl. Franchi (2013) $72 f$.

14 Die Personifikation des Flusses wird auch auf stilistischer Ebene unterstützt: Der in Vers 144 erfolgende Subjektwechsel von Dionysos zu Paktolos stellt eine Verbindung zwischen beiden Figuren her, Wein- und Flussgott werden wie selbstverständlich in einem Atemzug genannt. 
Und die Satyrn warfen in ihrem Spiel die Füße in die Luft und sprangen kopfüber in den Fluss. (150) Einer von ihnen schwamm, von selbst schwebend, mit den Händen rudernd und mit dem Kopf in den Wellen. Und er zog eine Spur durch die Wasserwogen und teilte mit nach hinten gewandten Füßen das reichlich fließende Wasser. - Wieder einer tauchte in die Tiefe, ganz nach unten ans Flussbett, und suchte am Grund nach der farbenprächtigen Fischbeute. (155) Er streckte blind seine Hand nach den schwimmenden Fischen aus, verließ die Tiefe wieder und reichte Bakchos die Fische, die gerötet waren vom Schlick des überreichen Flusses. - Und Silenos presste, die Beine eng aneinander, die Füße zusammen und trat als leichtfüßiger Springer in den Wettstreit mit einem Satyrn: (160) Er sprang mit dem Kopf voran, sich um die eigene Achse drehend, in den Strom, von oben in die weite Tiefe, und streifte mit seinem Haar den Schlamm. Die beiden Füße bohrte er in den glitzernden Morast und schürfte nach dem Kieselreichtum des Flusses. - Und ein anderer streckte mitten in den Fluten seinen Rücken in die Luft, (165) ließ seine Schultern unbenetzt und tauchte bis zu den Hüften ins Wasser, tief im Nass stehend, regungslos. Er spitzte seine bloßen Ohren, benetzte seine haarigen Beine im glitzernden Strom, und sein ihm natürlich gewachsener Schweif schlug mit einer Drehbewegung das Wasser.

Sowohl das Kollektiv der Satyrn (Nonn. D. 10,148f.) als auch die einzelnen Individuen (150-152, 153-157, 158-163, 164-168) sind Gegenstand einer Textpassage, die mit der sprachlichen Umsetzung von Bewegung und Dynamik den Aktionsraum der Erzählung definiert, ${ }^{15}$ die eher statisch gehaltene Ekphrasis der Flusslandschaft schlägt um in eine Ekphrasis von Handlungen, die den konkreten Ort, an dem sie sich abspielen, beleben und diesen unmissverständlich als dionysisch etikettieren. Die Wassersprünge und Tauchgänge der Satyrn erscheinen auf den ersten Blick befremdlich und ungewöhnlich - vergleichbare Bade- und Schwimmszenen sind in der antiken Literatur nur selten zu finden. ${ }^{16}$ Nonnos' Vorliebe für intensive Bewegung und Gestik wie an dieser Stelle lässt zeitgenössische Erfahrungen sichtbar werden, so gehören szenische Vorführungen in Gestalt von Pantomimen zu den Alltagserfahrungen des spätantiken Publikums und begegnen auch an anderen Stellen in den Dionysiaka. ${ }^{17}$ Die verspielten Sprünge nehmen zudem bereits die Rolle der Satyrn als Kultbegleiter des Dionysos vorweg, die am Ende der Ampelos-Episode erstmals ihre volle Ausformung erhält: Die wilden Bewegungen beim Spiel im Fluss stehen stellvertretend für den ausgelassenen kultischen Tanz der Anhängerschaft des Weingottes. ${ }^{18}$

Zum vollen Verständnis der Szene tragen die folgenden Verse bei, in denen der Erzähler zu Dionysos und der Frage nach seiner Wesenheit Stellung bezieht (Nonn. $D$. 10,169-174):

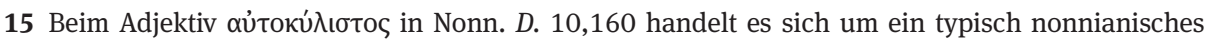
aỦto-Kompositum, das sich mit Ausnahme von Opp. C. 2,289, Opp. H. 2,604 und Georgios Pisides, De vita humana 90 nur bei Nonnos findet, vgl. etwa Nonn. D. 10,356, 376; 11,89, 218, 478; P. 11,112; 21,31. Zu den aỦंo-Komposita vgl. Kap. 2.5 Anm. 35.

$16 \mathrm{Zu}$ den Schwimmszenen in den Dionysiaka und zu möglichen Vorbildern siehe Böhm (1948) 65166; Kröll (2013).

17 Vgl. etwa den Tanz Marons in Nonn. D. 19,214-218.

18 Zur Bewegung der Satyrn in der bildenden Kunst der Antike vgl. Zanker P. (1998) 30-38. 


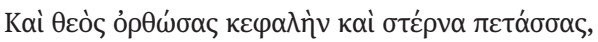

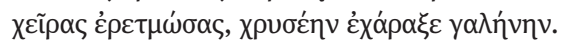
170

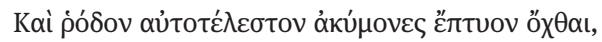

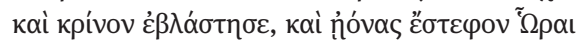

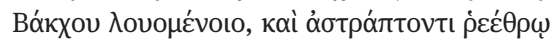

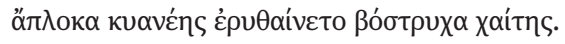

Und der Gott richtete sein Haupt auf und machte seine Brust breit, (170) und durchschnitt mit den Händen rudernd die goldglatte Wasseroberfläche. Und die von den Wellen unberührten Flussbänke brachten spontan Rosen hervor, Lilien blühten auf, und die Horen bekränzten die Ufer, als sich Bakchos badete, und im gleißenden Strom röteten sich die ungeflochtenen Locken seines schwarzen Haares.

Nach dem Intermezzo mit den schwimmenden und tauchenden Satyrn rückt Dionysos erneut ins Zentrum, indem er einmal mehr nicht nur die Hauptfigur, sondern auch das Hauptthema der Episode darstellt. Den entscheidenden Hinweis dafür liefert die Benennung als $\theta$ cós in Vers 169. Der Dichter beantwortet die Frage, ob es sich beim unehelichen Zeus-Sohn nun tatsächlich um einen Unsterblichen handeln könne, schon zu Beginn positiv, der Vorverweis auf Dionysos' Göttlichkeit könnte also nicht eindeutiger sein. Das Bad im Paktolos ist göttliche Epiphanie; ${ }^{19}$ der locus amoenus, der sich aus der explosionsartigen Ausbreitung der Natur in der Umgebung des Paktolos ergibt, ist deren Äußerung. Eine Bestätigung seines göttlichen Status erlangt Dionysos zudem durch die Anwesenheit der Horen, der Göttinnen der Jahreszeiten, denen in der Komposition der Ampelos-Episode eine besondere Funktion zukommt: ${ }^{20}$ Die Metamorphose des Ampelos in einen Weinstock vollzieht sich erst nach deren offiziellem Bittgang zu Helios (Nonn. D. 12,1-117). Gestaltung, Zweck und Ziel der Eröffnungsszene werden transparent gemacht: Es geht nicht nur um die Einführung von Hauptschauplatz und Protagonisten, sondern auch um die Einordnung der Hauptfigur Dionysos in die Reihe der anerkannten Gottheiten, die über ihre Kultlandschaft und ihre kultischen Anhänger verfügen.

\subsection{Kompositionsprinzipien und narratives Ziel}

Mit der Eingangsszene bezieht Nonnos nicht nur Stellung hinsichtlich seiner Figuren und definiert den Ort des Geschehens, er liefert auch Indizien für ein tief greifendes Verständnis seiner Dichtung. Es finden sich mehrere Anhaltspunkte für narrative Kompositionslinien und intratextuelle Bezüge, Figuren und Ort sind von Beginn der Episode an auf ihr Ende hin ausgerichtet. Worauf in der Erzählung über den badenden Gott und seine Anhängerschaft besonderer Wert gelegt wird, taucht an späterer Stelle

19 Zu Dionysos als Gott der Epiphanie vgl. Henrichs (2011).

20 Die Horen sind in besonderer Weise mit dem Schicksal des Dionysos verbunden: Erstmals bei dessen Geburt aus dem Schenkel des Zeus anwesend, begleiten sie ihn auf den wichtigsten Stationen seines Lebens; zu den Horen vgl. S. 180-197 und Kap. 7.3. 
wieder auf, und insbesondere das Wasser und die dionysischen Wirkkräfte sind für die gesamte Episode konstitutiv.

In mehreren Szenen der Ampelos-Episode wird Wasser zum Handlungsträger: neben dem Spiel der Satyrn im Paktolos auch in den Schwimmkonkurrenzen zwischen Dionysos und Ampelos (Nonn. D. 11,1-55) sowie zwischen Kalamos und Karpos (400426). ${ }^{21}$ Das spielerische Fischen der Satyrn in den Fluten des Paktolos bereitet die Schwimmwettbewerbe vor, die vom flüssigen Element geprägte Erzähllinie wird bis ans Episodenende weitergezogen, wenn die Schlussszene wiederum Dionysos in Begleitung seiner Entourage zeigt, welche nun nicht mehr mit Wasserspielen, sondern mit der ersten Weinkelterung und -verkostung beschäftigt ist (Nonn. D. 12,328-339). Das Element Wasser findet sich auch in der Szene, in der der junge Satyr vom Stier mit Wasser besprengt wird, und nimmt die erforderliche Bewässerung der Weinstöcke sowie Ampelos' soteriologische Rolle vorweg (Nonn. D. 11,161-166). ${ }^{22}$ Bildet zu Beginn der Ampelos-Episode noch das Wasser die Projektionsfläche für das unmittelbare Geschehen, so übernimmt diese Funktion am Ende das Getränk des neuen dionysischen Zeitalters, der Wein, der das Wasser durch das dionysische Element par excellence ablöst. Die Genese des vergorenen Traubensaftes, das Ergebnis der zweieinhalb Bücher umfassenden Ampelos-Episode, bildet die Voraussetzung für die Ausstattung des Protagonisten mit seinem wirkungsmächtigen Attribut, das ihn für sämtliche folgenden Geschehnisse rüstet und ihm hilft, sich in den Kämpfen gegen die Inder zu profilieren.

Schon das Wasser der Eingangsszene verweist auf den Ausgang der Episode, handelt es sich doch um den Fluss Paktolos, dessen dionysischer Esprit von Anfang an den Kern der Erzählung bildet. Diese dionysische Komponente verknüpft die AmpelosEpisode mit dem vorhergehenden Sprung Inos ins Meer: ${ }^{23}$ Inos Beweggrund, ihr irrationaler und unkontrollierbarer Wahnsinn, ist ebenso der dionysischen Sphäre zugehörig wie der Fluss Paktolos in Lydien; hier wie dort ist das Wasser aufs Engste mit

21 Das Element Wasser ist nicht nur für die Ampelos-Episode von herausragender Bedeutung, es stellt auch in anderen Szenen der Dionysiaka ein wesentliches Gestaltungsprinzip dar, etwa in den Badeszenen des Aktaion (Nonn. D. 5,304-325, 476-488), der Persephone (5,585-589, 601-610), der Semele (7,171-279), des Morrheus (35,185-191), der Klymene (38,116-129) und der Artemis (48,302-375). Außerdem ist Wasser auch für zwei große Erzähllinien des Epos bestimmend: die von Zeus geschickte Sintflut im 6. Buch und die Kämpfe des Dionysos-Heeres gegen die Inder am Fluss Hydaspes in den Büchern 22-24; vgl. auch den Meergreis Proteus im Proömion. Zur narrativen Funktion von Wasser in der Ampelos-Episode und in den Dionysiaka siehe Fauth (1981) 45-49; Newbold (2001); Frangoulis (2008); Kröll (2013); zu den Personifikationen von Wasser in den Dionysiaka vgl. Miguélez Cavero (2013) 365-372. - Ähnlich symbolische Bedeutung tragen Wasser, Milch und Honig im Zusammenhang mit der Beroe-Figur in Nonn. D. 41,155-262: Auch Beroe wird durch ein Bad (in der Hippokrene [41,225-227]) in ihre künftige Rolle gleichsam initiiert, vgl. Accorinti (1997). Zur Verbindung von Dionysos mit dem Element Wasser vgl. auch Daraki (1985) 34-41.

22 Vgl. Gigli Piccardi (2003a) 372f. - Ein für die Bedeutung der vorliegenden Szene interessanter Aspekt liegt in der Tatsache, dass der Täter (der Stier) am Opfer (Ampelos) gleichsam als umgekehrte Opferszene die kultischen Handlungen vollzieht, indem er dieses mit Wasser besprengt.

23 Vgl. Fauth (1981) 45f. 
Dionysos verbunden, ${ }^{24}$ dessen Göttlichkeit nicht nur den Hintergrund für die Erzählung bildet, sondern in Gestalt der wahnsinnigen Ino und des personifizierten Paktolos manifest und materialisiert wird. Wie Inos Wahnsinnstat im 10. und der Wein am Ende des 12. Buches stellt auch der Paktolos mit seinem Wasser ein unverkennbares Charakteristikum des Dionysischen dar. Über die zweieinhalb Bücher der Ampelos-Episode verteilt werden immer wieder dionysische Kultelemente sichtbar gemacht und in die Handlung integriert. Zahlreiche erzählerische Details sind auf das Ziel, die Initiierung des dionysischen Kultes, ausgerichtet, der in seiner vollen Ausprägung erst mit dem Ende des 12. Buches existieren kann. Die Verwandlung von Wasser in Wein ist gleichzeitig ein wichtiger Emanzipationsprozess für Dionysos in Richtung Anerkennung als legitime Gottheit, der sich von seiner Epiphanie im 10. Buch bis zu seiner Apotheose am Schluss der Dionysiaka vollzieht: Ausgangs-, Mittel- und Endpunkt der Ampelos-Episode ist der Weingott Dionysos.

Dabei darf der hart anmutende und künstlich erscheinende Übergang von der panisch reagierenden Ino zu der idyllisch anmutenden Genreszene mit den badenden Satyrn als vom Dichter bewusst gesetzt verstanden werden. Ein wesentliches Merkmal der narrativen Technik des Nonnos ist es, Kontinuität bei gleichzeitiger Episodenhaftigkeit zu schaffen: Wie jeder Ependichter will er eine stringente, durchgehende Geschichte erzählen, andererseits aber innerhalb seines kaum überschaubaren GroßEpos einzelne mehr oder minder miteinander verbundene Episoden herauslösen, denn diese Erzählungen innerhalb der Erzählung erlauben es ihm, seinen Erzählgegenstand in ein außerordentlich breites Spektrum aufzufächern: Sowohl die Ino- als auch die Ampelos-Episode könnten für sich allein genommen eine eigene Kleindichtung bilden. ${ }^{25}$ Nonnos steht mit diesem mosaikartigen Stil in der Tradition der Spätantike und ihrer künstlerischen Ausdrucksformen, so finden sich etwa in den kaiserzeitlichen Mosaikzyklen, die mehrere Einzelszenen zu einem großen, übergeordneten Thema zusammenfassen, gleichzeitig jedoch stets deren Unabhängigkeit vom Leitsujet wahren, Beispiele für ein derartiges ästhetisches Programm. ${ }^{26}$

Wesentliche thematische Linien, die sich durch die Episode ziehen und diese zusammenhalten, sind neben dem Element Wasser die dionysischen Wirkkräfte, die als narrative Klammer vom Anfang im 10. bis zum Ende im 12. Buch fungieren: ${ }^{27}$ Die Epiphanie des Dionysos, die grünende Vegetation und die Anwesenheit der Horen deuten auf den End- und Höhepunkt der Episode voraus, die Genese des Weines, die Zuschreibung der neuen Pflanze und die damit einhergehende Einordnung des Dionysos in die Riege der olympischen Götter. Dionysos und das Dionysische spielen auch im Verlauf der Ampelos-Erzählung eine wichtige Rolle, wenn etwa Ampelos infolge

24 Zur Verbindung des Wassers mit Dionysos vgl. Merkelbach (1988) 109-111; Forbes Irving (1990) 193; Kerényi (1994) 119f., 180f.; Giraudet (2005) 77.

25 Zur Epyllien-Theorie vgl. D’Ippolito (1964) und S. $12 \mathrm{f}$.

$26 \mathrm{Zu}$ den Gemeinsamkeiten nonnianischen Dichtens und spätantiker bildender Kunst vgl. S. 247249.

27 Zum Dionysischen als Kompositionsmittel vgl. Kap. 7.4. 
seines dreifachen Sieges über Dionysos in den sportlichen Wettkämpfen in Maßlosigkeit schwelgt und sich wie der Gott Dionysos selbst kleidet und gibt (Nonn. D. 11,5670), oder wenn er den Stier gleichsam wie ein Opfertier für Dionysos schmückt und damit seinen Tod und seine Metamorphose verursacht (155-184). Mit der Badeszene greift Nonnos zudem auf ein früheres Ereignis in der Biographie des Gottes zurück: das Bad des Neugeborenen durch die Nymphen, das er an der zu erwartenden Stelle im 9.

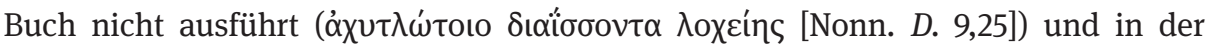
Ampelos-Episode nachträgt. ${ }^{28}$

Die Schwimm- und Badeszene zu Beginn der Episode liefert nicht nur hinsichtlich ihrer Thematik, sondern auch in ihren sprachlichen Details Vorverweise auf das narrative Ziel. Obwohl in der vorliegenden Textstelle Metamorphose, Weinstock und Wein nirgendwo explizit genannt sind, setzt der Dichter bewusst gewählte Signalwörter. Dionysos ist zum Zeitpunkt der Ereignisse zu einem Jugendlichen „erblüht“

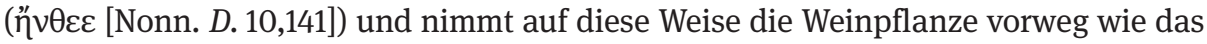

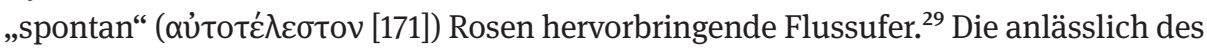
Bades des Dionysos aufblühende Natur, symbolisiert durch Rosen und Lilien ( [Nonn. D. 10,171], кpívov [172]), verweist auf die zukünftige Weinrebe. Das Adjektiv

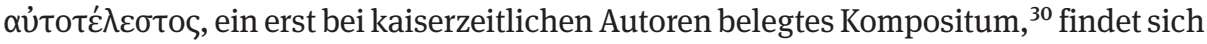

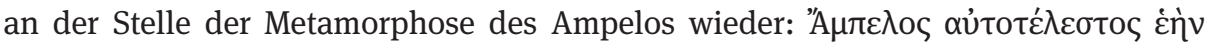

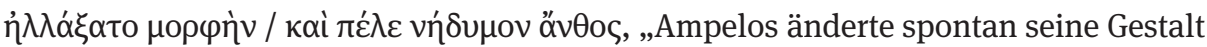
und wurde zu einer reizvollen Blume“ (Nonn. D. 12,175). Wie auf die neue Pflanze wird auf die Trauben und auf das daraus gewonnene Getränk, den Wein, verwiesen. Die Rose impliziert die rote Farbe der Weintrauben wie „die purpurnen Sande“ (лорцu-

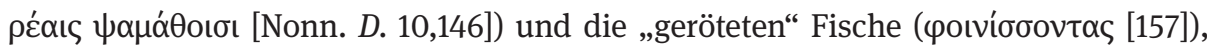

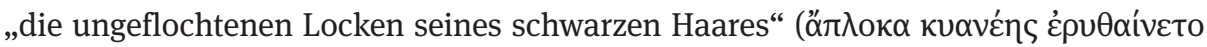

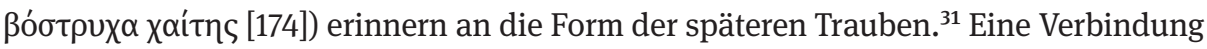
von Ampelos' Haarlocken mit dem Aussehen der Weintrauben erfolgt gleichfalls in der Metamorphose im 12. Buch, wo in der detailreichen Schilderung auf die Verwandlung einzelner Körperteile des Satyrn, darunter auch auf das zu Trauben werdende Haar,

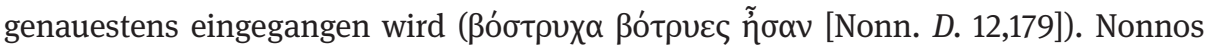
gestaltet seine Erzählung demnach auch auf der lexikalischen Mikroebene bewusst im Hinblick auf das angestrebte narrative Ziel.

$28 \mathrm{Zu}$ den literarischen und ikonographischen Zeugnissen zum ersten Bad des Kindes Dionysos und zu den Parallelen im Achill- und Alexander-Mythos sowie in der Taufe Jesu vgl. Bowersock (2011).

29 Nonnos zeigt eine Vorliebe für Adjektive und Epitheta, die Wachsen und Bewegung ausdrücken, vgl. Lindsay (1965) 377.

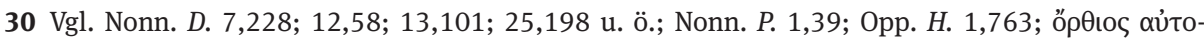

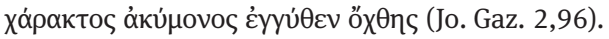

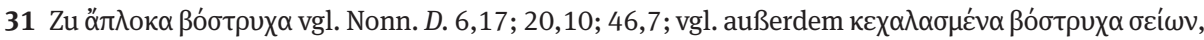

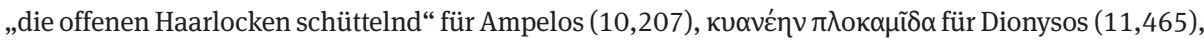

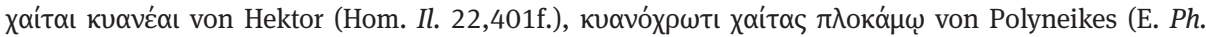

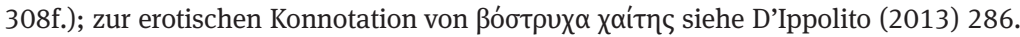




\subsection{Literarische Technik, Sprache und Stil}

Ein Hauptinteresse liegt für Nonnos auch in der Vermittlung visueller Eindrücke. In der Eingangsszene der Ampelos-Episode wird vornehmlich durch die breite und wortreiche Deskription des Sonnenlichts (Nonn. D. 10,141-144), des golden schimmernden

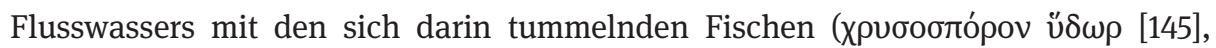

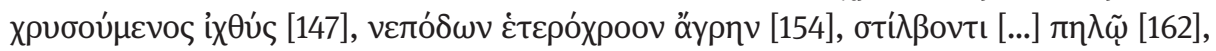

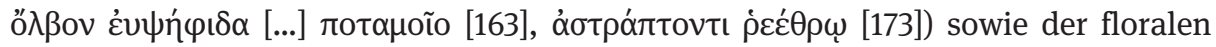
Pracht der Ufervegetation (171-174) ein imaginäres Gemälde vor dem geistigen Auge des Lesers erzeugt. Die Reprise derselben optischen Eindrücke in stets veränderter Wortwahl ist Teil des nonnianischen Dichtungsprogramms, welchem Nonnos mit der

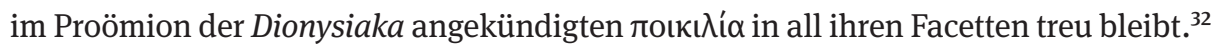
In dem von ihm bevorzugten parataktischen Satzbau lässt er Bild auf Bild folgen, für ein und denselben Sachverhalt bietet er eine Vielfalt an sprachlichen Umsetzungsmöglichkeiten. ${ }^{33}$ Allein für die Begriffe Fluss, Strömung, Strom und dergleichen liefert er insgesamt zwölf verschiedene lexikalische Äquivalente, einige werden sogar

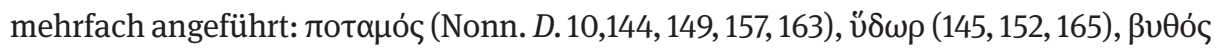

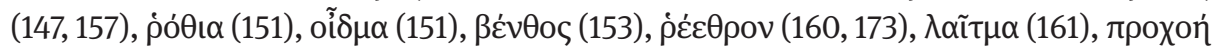

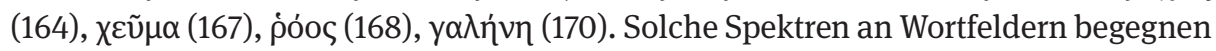
allenthalben in der Ampelos-Episode. Die permanente Variation als dichterisches Gestaltungsmittel stellt einen wesentlichen Gesichtspunkt des spätantiken Kunstschaffens dar, wobei die Beschäftigung mit einem Thema aus einem immer wieder veränderten Blickwinkel und mit voneinander geschiedenen, aber dennoch entsprechenden Sprachmitteln im Dienst der poetischen Ausdruckskraft steht.

Von besonderer Relevanz im Hinblick auf die literarischen Gestaltungsprinzipien nonnianischer Epik ist auch die Inszenierung von Bewegung und Gesten: ${ }^{34}$ Mit minutiöser Liebe zum Detail werden die einzelnen Bewegungsphasen der im Wasser schwimmenden und tauchenden Satyrn geschildert, die durch eine ganze Reihe von

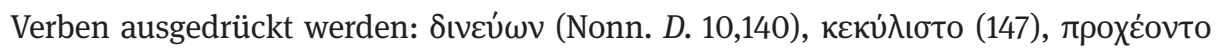

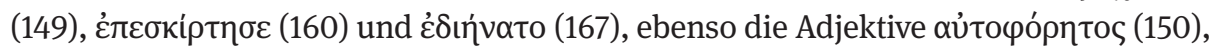

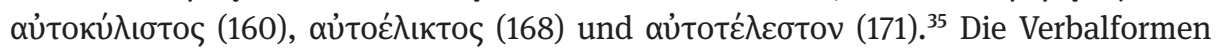

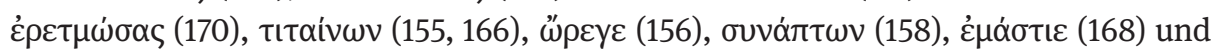

32 Zu Proteus und zum Proömion der Dionysiaka vgl. Gigli Piccardi (1993); Agosti (1996); Newbold (2001) 170; Bannert (2008).

33 Zum Stilprinzip der Wiederholung in Nonnos vgl. Schmiel (1998a).

34 Vgl. Friedländer (1931) 44f.; Miguélez Cavero (2009).

35 Komposita mit dem Präfix aỦंo- drücken in den Dionysiaka stets spontane Bewegung aus, vgl. etwa in der Szene mit dem pantomimischen Tanz des Silenos anlässlich der Leichenspiele zu Ehren des

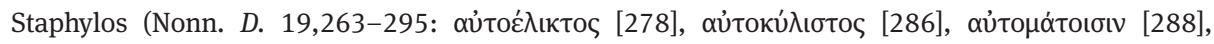

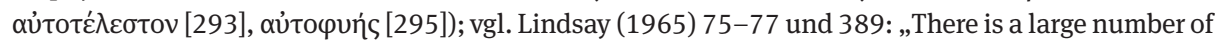
auto-terms, which bring out how deeply rooted in Nonnos' worldview was the idea of spontaneous inner movement as an aspect of Dionysiac liberation.“ 


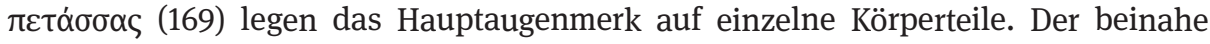
vollständige Katalog von Körperpartien vom Kopf bis zu den Füßen macht die agie-

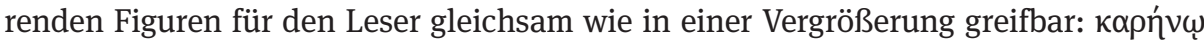

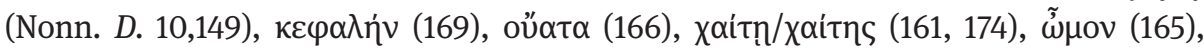

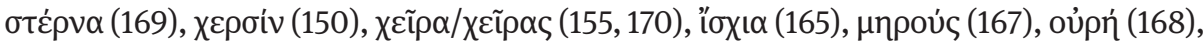

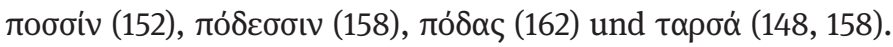

Die schwungvollen Bewegungen von Dionysos und seinen Begleitern, die sich im Zappeln der Fische und im Emporwachsen der Pflanzen fortsetzen, sind auch in den folgenden Szenen ein zentrales Gestaltungselement. Sowohl in den sportlichen Wettkämpfen zwischen Dionysos und Ampelos, welche schon allein aufgrund ihrer Thematik für die sprachliche Darstellung von Bewegung prädestiniert sind, als auch in seinen Figuren-Porträts, für die vorschnell und auf den ersten Blick eine gewisse Statik vermutet werden könnte, erweist sich Nonnos als Meister in der sprachlichen Umsetzung von visuellen und gestischen Eindrücken. So wird etwa die Ekphrasis der vier Jahreszeiten aufgrund der sprachlichen Umsetzung von Bewegung gleichsam zu einem kleinen Drama innerhalb der epischen Erzählung (Nonn. D. 11,485-521). ${ }^{36}$ Die Visualisierung, die durch das Mittel der epischen Sprache erfolgt, wird zu einem entscheidenden kompositionellen Instrument der Dionysiaka. Das nonnianische Epos erlangt seine ästhetische Wirkung über das Veranschaulichen, das unmittelbare VorAugen-Führen von Figuren der Handlung sowie über die besondere Akzentuierung von Bewegungsabläufen. Ein signifikantes Merkmal des spätantiken Epos ist, dass sich im Unterschied etwa zum klassisch-homerischen Epos - die beschreibenden Partien, die Ekphrasen von Orten und Figuren, gewissermaßen zu eigenberechtigten narrativen Einheiten entwickeln; insofern steht es in direkter Nachfolge hellenistischer Dichtungsformen. ${ }^{37}$ Durch die Artikulation von Visuellem und Gestischem, und zwar in ganz unterschiedlichen narrativen Kontexten, erzeugt der Dichter eine Kette von aufeinanderfolgenden Einzelszenen, die, von den großen erzählerischen Linien weitgehend abgekoppelt, zu unabhängigen narrativen Größen werden. ${ }^{38}$ Dies hat nun nicht zur Folge - wie vor allem am Beginn der Forschungen zu den Dionysiaka konstatiert -, dass diese jeglicher szenenübergreifender Kompositionslinie entbehren. War es in Ilias und Odyssee noch das Ursache-Wirkungs-Prinzip, das einzelne Szenen und Ereignisse zu einem großen epischen Ganzen verband - menschliches wie göttliches Handeln zieht logisch nachvollziehbare Konsequenzen nach sich - und den kom-

36 Vgl. S. 180-187.

37 Zum Faktor Bewegung in antiken Ekphrasen vgl. Wandhoff (2003) 54-57, der als besonderes Charakteristikum der hellenistischen Dichtung die „kinetische Aufladung der Ekphrasen“ (55) sieht; vgl. außerdem Beaujour (1981) 33: „Static ,figures“ spring into action: this effect is analogous to the animation of a motion-picture freeze shot"; siehe auch Schönberger (1995) 158, der Ekphrasis als „belebte Beschreibung“ charakterisiert und die Darstellung von Bewegung als eines der Mittel der Bildbeschreibungen Philostrats hervorhebt (170); vgl. auch Arnulf (2004) 28; Webb (2009) 187-190. 38 Vgl. Riemschneider (1957) 69: „Wie auf einer Perlenschnur reiht der Dichter Bild an Bild, das Verbindungsglied zwischen den einzelnen Erzählungen ist kein logisches, es ist rein assoziativ.“ 
positionellen Rahmen bestimmte, sind es in den Dionysiaka wiederkehrende Erzählmuster und narrative Techniken, die Verbindungslinien zwischen den Einzelszenen ziehen. Der sprachlichen Umsetzung von visuellen Eindrücken kommt dabei eine entscheidende Rolle zu: Das Bild, das die Leserschaft vom Paktolos-Fluss, von den schwimmenden Satyrn und dem badenden Dionysos erhält, soll sie auf künftige Ereignisse in der Ampelos-Episode vorbereiten. Beschreibung und Bewegung werden zu Handlungsträgern einer neuen, spätantiken Spielart des Epos, wobei es Ziel dieser epischen Narration ist, die Bewegung und das orgiastische Wesen des Gottes mit sprachlichen Mitteln zum Ausdruck zu bringen. Die im Zeichen des Dionysos stehenden Dionysiaka werden zum Sinnbild eines Gottes, der wie kein anderer der Olympier für permanente Veränderung steht, ${ }^{39}$ das gesamte Epos ist von dionysischem Taumel durchwirkt und von der spezifisch dionysischen Art zu dichten geprägt. ${ }^{40}$

Die Verankerung der Dionysiaka im Kontext der Spätantike erfolgt jedoch nicht nur über die großen Kompositionslinien des Epos, Nonnos lässt auch auf der sprachlichen Mikroebene erkennen, dass sein Werk mehr als tausend Jahre nach Homer entstand.

Ein unverkennbares Charakteristikum nonnianischen Dichtens stellen das erlesene Vokabular und die zahlreichen Neologismen dar. Der außerordentliche lexikalische Reichtum und die Vielschichtigkeit der nonnianischen Sprache veranlassten W. H. D. Rouse in seinem Vorwort zu Band III der Loeb-Edition, den Stil der Dionysiaka als „Niagara of words“41 $\mathrm{zu}$ charakterisieren. Allein in den ersten Versen der AmpelosEpisode finden sich zwei hapax legomena der griechischen Sprache, хрvбобтópos

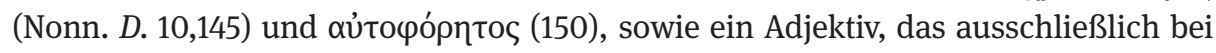

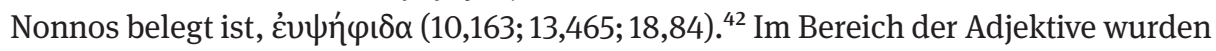
allein 375 hapax legomena nachgewiesen, Wörter, die ein oder mehrmals, jedoch immer ausschließlich bei Nonnos zu finden sind, außerdem 115 Adjektive, die erstmals bei Nonnos belegt sind. ${ }^{43}$ Durch diese lexikalischen Neuschöpfungen zeigt sich der Dichter in höchstem Maße als Innovator der griechischen epischen Sprache, die seit Homer einen weiten Weg zurückgelegt hat, wobei sich Nonnos jedoch nicht vollständig

39 Vgl. Duc (1990) 191.

40 Zur dionysischen Rauschhaftigkeit als Stil- und Kompositionsprinzip vgl. Lesky (1971): „Die Dionysiaka sind bei allem Reichtum motivischer und formaler Voraussetzungen doch ein Werk eigenen Gepräges. Sie sind es durch den Zug dionysisch-rauschhafter Erregung, der durch das Ganze geht“; Schmid u. Stählin (1980/61924) 965: „der taumelnde Überschwang der nonnianischen Dichtung“; Fauth (1981) 33f.; Shorrock (2001) 207-213; Hernández de la Fuente (2008) 54.

41 Rouse Bd. 3, VI. - Schon Villoison (1782) 7f. charakterisiert den Stil des Nonnos folgendermaßen: [...] turgidi quidem et ampullis, canoris nugis et verbis sesquipedalibus ad nauseam pleni, semper licentia

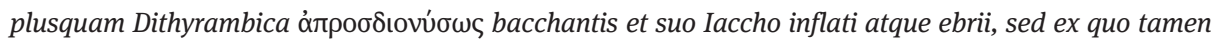
multa erui possunt ad Mythologiam reconditam et pleniorem antiquitatis notitiam, quae frustra in alio scriptore quaererentur. Zum Stil vgl. auch Golega (1930) 28-62.

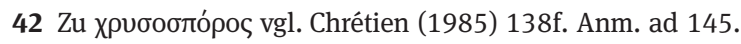

43 Vgl. Espinar Ojeda (2002). - Zu Nonnos' Vorliebe für Adjektive und Epitheta vgl. Golega (1930) 4955; String (1966) 101-108; Livrea (1989) 58-60; Greco (2004) 35-37; Miguélez Cavero (2008) 114121; Franchi (2013) 197-202. 
von seinen Vorbildern emanzipiert, sondern sich durchaus in die Tradition hellenistischer und kaiserzeitlicher Dichtung stellt. Einen Teil seines Wortschatzes übernimmt

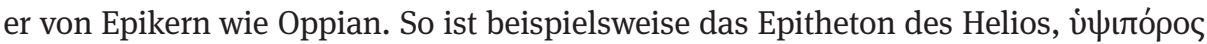
(Nonn. D. 10,141), zum ersten Mal in den Kynegetika belegt und findet sich auch bei Dichtern nach Nonnos. Im Unterschied zum vereinzelten Erscheinen bei den genannten Autoren schöpft Nonnos jedoch aus dem Vollen und setzt das Adjektiv über

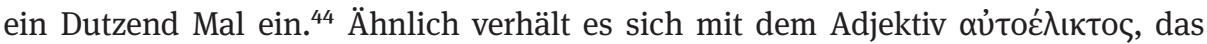
sowohl in den Dionysiaka als auch in der Paraphrase mehrmals zu finden ist, sonst jedoch nur je einmal bei Johannes von Gaza und Christodoros von Koptos. ${ }^{45}$

Die häufige Setzung von seltenen, komponierten Adjektiven in variierendem Kontext ist ebenso eine Besonderheit nonnianischer Sprache wie die Bevorzugung eines Nominalstils, der die Hauptaussage eines Satzes vornehmlich in die Substantive, Adjektive und Partizipien legt. Nicht nur die Metrik des Nonnos, sondern auch seine Sprache und sein Stil hatten Breitenwirkung in der Spätantike. Dichter, die gemeinhin der sog. Schule des Nonnos zugerechnet werden, etwa Musaios, Kolluthos, Pamprepios oder auch Paulos Silentiarios und Johannes von Gaza, übernehmen diese neuen Tendenzen in ihre eigenen Werke und prägen das Kunstverständnis ihrer Zeit. ${ }^{46}$

\subsection{Nonnos' Umgang mit den Quellen}

Ähnlich innovativ wie in Metrik und Lexik erweist sich Nonnos, der „segundo Homero“47 und „latter-day Homer“48, auch in der Verarbeitung seiner literarischen Referenztexte. Homer, Archeget der epischen Dichtung, steht für viele Stellen der Dionysiaka Pate, ${ }^{49}$ auch in der Szene um die badenden Satyrn ist sein Einfluss spürbar, und zwar in einigen Wendungen, die von homerischer Diktion inspiriert sind: Der

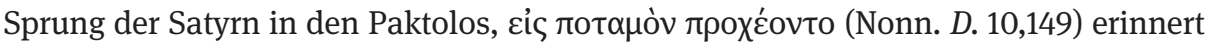

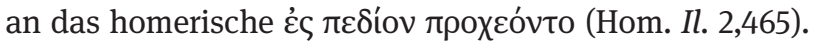

Neben einzelnen Wörtern werden auch feststehende Wendungen sowie epische Topoi und Motive, die aus Ilias oder Odyssee geläufig sind, in die Dionysiaka trans-

44 Vgl. Nonn. D. 2,126, 575; 3,218; 6,107; 9,308; 16,285; 22,42; 23,118, 301; 36,325; 37,729;

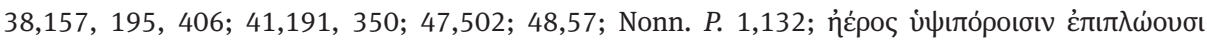

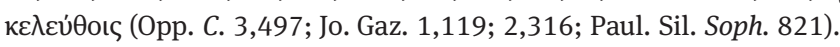

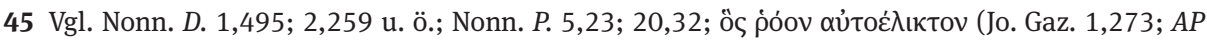
2,1,269 [Christodoros von Koptos]).

46 Zur Schule des Nonnos vgl. Mommsen (1895) 244-246; Lindsay (1965) 362; Braden (1978) 7080; Trypanis (1981) 395-402; Whitby (1994); Hernández de la Fuente (2008) 29 und 37; Miguélez Cavero (2008) 85-105, 188-190, 371-382. Zum Stil des Musaios und des Nonnos vgl. Gelzer (1967) und (1968); siehe auch S. 256.

47 Hernández de la Fuente (2008) XI.

48 Egberts u. a. (2002) VII.

$49 \mathrm{Zu}$ Homer in der Ampelos-Episode siehe Kap. 5.2. 
feriert und oft in einen gänzlich neuen Kontext gestellt. ${ }^{50}$ Die altbekannten Inhalte werden nicht nur dem Sujet der Dionysiaka angepasst, sondern lassen auch die Referenzquelle Homer in neuem Licht erscheinen. Szenen, die sich lediglich einmal bei Homer finden, etwa der Schiffs- und Troerkatalog im 2. Gesang der Ilias, der Flusskampf, die Schildbeschreibung und die Leichenspiele, werden von Nonnos verdoppelt (der Heereskatalog in Nonn. D. 13 und $14^{51}$ ), verdreifacht (die Spiele in den Büchern 10, 11, 20 und 37) oder in Gestaltung und Umfang deutlich erweitert (der Flusskampf in Nonn. D. 23-24 sowie 39-40). Charakteristische Bilder wie das Sperlings-Prodigium im siebten Kriegsjahr sind aus Homer entlehnt (Nonn. D. 25,1-10) (22 $^{2}$ und werden durch die Kontextualisierung im Rahmen des Dionysos-Mythos auf einer metapoetischen Ebene reflektiert. Die Dionysiaka sind keine bloße Mythensammlung um den Gott Dionysos, sondern eine aktive Auseinandersetzung mit den Möglichkeiten epischen Dichtens. Die Art und Weise, wie Nonnos mit seinem Vorbild Homer umgeht, wird durch den Terminus „imitatio cum variatione ${ }^{\text {“53 }}$ treffend charakterisiert. Der spätantike Dichter

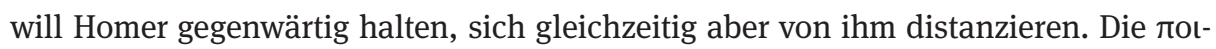

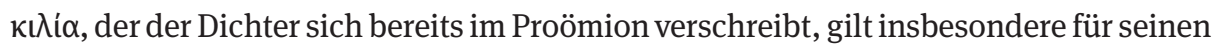
Umgang mit der Richtschnur jeglichen epischen Schaffens, den Werken Homers.

Meist weniger eindeutig als bei Homer lassen sich Einflüsse der hellenistischen Dichtung auf Nonnos festmachen. Ausgewählte sprachliche Anklänge an hellenistische Autoren wie Kallimachos, Arat, Apollonios Rhodios und Theokrit sind über die gesamten Dionysiaka verteilt. Die Anspielungen erfolgen stets auf äußerst subtile Weise, und Nonnos lässt häufig offen, ob es sich nun tatsächlich um bewusst gewählte Zitate handelt, die den inhaltlichen Rahmen des Bezugstextes in die Dionysiaka hereinnehmen wollen, oder ob diese vom Kontext des Referenztextes weitgehend losgelöst als bloße Sprachspiele ihre Wirkung erzielen. Beispiele dafür sind auch in der ersten Szene der Ampelos-Episode zu finden.

In Nonn. D. 10,143 verschränkt der Dichter offenbar zwei Zitate hellenistischer Autoren miteinander und bringt diese in den neuen Kontext um die badenden Satyrn

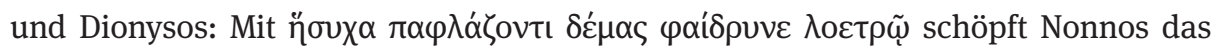
Wortmaterial sowohl aus Theokrit als auch aus Apollonios Rhodios: ${ }^{54}$ Im 6. Idyll des Theokrit wird Galateia, die Geliebte Polyphems, ,an der ruhig plätschernden Küste

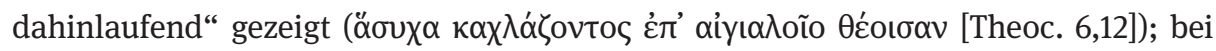
Apollonios erfreuen sich die in Kolchis angekommenen Argonauten und Phrixos-

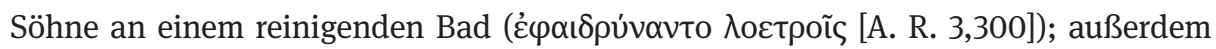
erteilt Medea Iason den Auftrag, sich mit dem von ihr zubereiteten Zaubermittel wie

50 Vgl. Wild (1885-1886) 3f.; Dihle (1989) 610.

$51 \mathrm{Zu}$ den Heereskatalogen in den Dionysiaka und zu ihrem Verhältnis zu Homer vgl. Chuvin (1991) 29-144.

52 Vgl. Wild (1885-1886) 45; Keydell (1932) 186f. (498f.); Schmid u. Stählin (1980/61924) 967. 53 Hernández de la Fuente (2008) 41.

54 Vgl. Chrétien (1985) 138 Anm. ad 143. Zum Versschluss vgl. auch Nonn. D. 7,241 u. 384; 16,7;

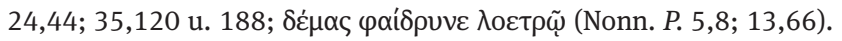


mit einem Öl einzusalben, um übermenschliche Kräfte zu erlangen und gegen die

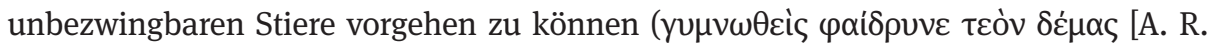
3,1043]). Als Gemeinsamkeit aller drei Referenzstellen ist auch die Liebesthematik festzumachen: Im Theokrit-Idyll ist es das Lied des Hirten Daphnis auf das Liebespaar Galateia und Polyphem, in den Argonautika die unerfüllte Liebe Medeas zu Iason; das Bad der Argonauten bei Apollonios erfolgt unmittelbar auf den Pfeilschuss des Eros auf Medea und das erste Aufflackern ihrer Liebe zu Iason. Somit wäre die Liebesbeziehung zwischen Dionysos und Ampelos, die sich am Flusswasser des Paktolos abspielt, mittels feinster intertextueller Verweise vorbereitet. Ein weiterer Aspekt tritt möglicherweise hinzu: die göttliche Unbesiegbarkeit, die Iason durch die Anwendung

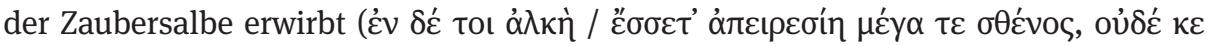

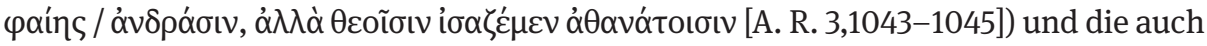
Dionysos und seine Begleiter, die Satyrn, auszeichnet. Die Frage, ob es sich beim jungen Dionysos tatsächlich bereits um einen Gott handelt, wird in den folgenden Szenen der Ampelos-Episode immer wieder thematisiert.

Ein weiterer Bezugstext für die Szene um die im Paktolos Badenden ist möglicherweise die Weltbeschreibung des Dionysios Periegetes. ${ }^{55}$ In den Versen 830-846 seiner poetischen Ausführungen zur antiken Geographie beschreibt er die Landschaft Lydien, die er wie Nonnos Maionien nennt (Mnovín [D. P. 830]) und durch das Tmolos-

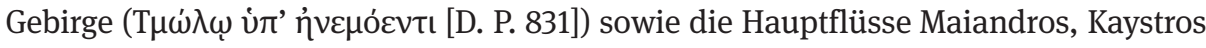
und Paktolos charakterisiert. Auffallend ist die Häufung von Vokabular, das auch bei Nonnos zu finden ist: Auch bei Dionysios „gurgelt“ der unzählige Goldpartikel füh-

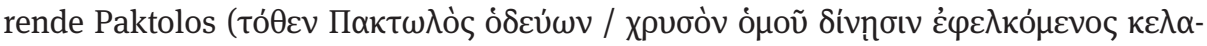

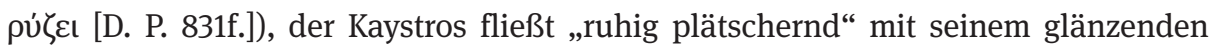

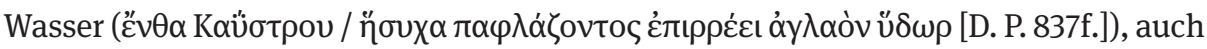
in dieser Umgebung wird Dionysisches sichtbar, und zwar in Gestalt der Mänaden, der Anhängerinnen des Dionysos (D. P. 839-845), zudem wird der Gott mit Namen genannt ( $\Delta$ เ $\omega v$ voı $[D$. P. 842]). Die Ähnlichkeit beider Stellen hinsichtlich der Lexik wird zusätzlich unterstützt durch den vergleichbaren Kontext. Obwohl die Weltbeschreibung des Dionysios als Quelle für die Eingangsszene der Ampelos-Episode möglich erscheint, ist Vorsicht geboten, da nicht in allen Fällen unstrittig ist, ob es sich bei der Verarbeitung einzelner Wörter und Wortgruppen tatsächlich um gezielte Zitate aus der hellenistischen und kaiserzeitlichen Literatur handelt. Insbesondere die AmpelosEpisode ist jedoch ein Beispiel dafür, dass es nicht immer bei bloßen lexikalischen Reminiszenzen bleiben muss, sondern dass Zitate und lexikalische Anlehnungen vom Dichter in Relation zum Erzählverlauf der Dionysiaka gestellt werden und so die Rezeption durch das Lesepublikum steuern können.

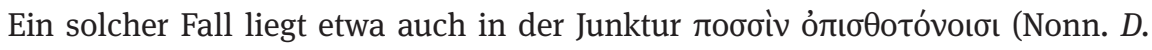
10,152) vor, welche sich in leicht variierter Form lediglich ein weiteres Mal in der 
griechischen Literatur wiederfindet, und zwar in den Phainomena Arats in Zusammenhang mit der Beschreibung der Sternbilder Krebs und Löwe (Arat. 147-151): ${ }^{56}$

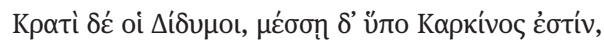

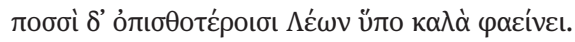

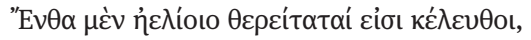

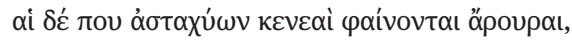

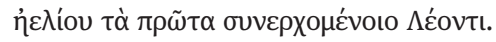

Zu ihren [sc. der Großen Bärin] Häupten sind die Zwillinge, mitten unter ihr der Krebs; unter ihren Hinterfüßen leuchtet der Löwe schön. Dort sind der Sonne heißeste Pfade. Leer von Ähren zeigen sich die Felder, wenn die Sonne zum ersten Mal im Löwen aufgeht.

Nonnos greift diese Stelle nicht zufällig auf: Das Sternbild Löwe steht für die erbarmungslose Sommerhitze (Arat. 149) und die Zeit der bereits abgeernteten Getreidefelder (Arat. 150). Unter ganz ähnlichen klimatischen Bedingungen läuft die Szene mit den badenden Satyrn und Dionysos ab, die sich zur heißesten Tageszeit im Wasser des Paktolos tummeln, wenn die Sonne den Zenit erreicht (Nonn. D. 10,141f.). Seit den Nonnos-Forschungen am Beginn des 20. Jhs. wurde auf die Bedeutung der Sternbilder und der astronomisch-astrologischen Angaben in den Dionysiaka verwiesen. ${ }^{57}$ Vor allem ein Sternbild wird sich als richtungsweisend für die Erzählung um Ampelos zeigen, die Jungfrau: $:^{58} \mathrm{Im}$ 12. Buch des Epos konsultieren die Horen die prophetischen Tafeln der Harmonia, die sich im Palast des Helios befinden (Nonn. D. 12,41-113). ${ }^{59}$ Die Aufschrift auf der dritten Tafel ordnet die Ampelos-Erzählung in einen größeren chronologisch-kosmologischen Kontext ein, wenn die Ankunft des Weines durch die Metamorphose des Ampelos angekündigt wird, und zwar zur Zeit der Sternbilder Löwe und Jungfrau (Nonn. D. 12,90-95); das unverwechselbare Charakteristikum dieses

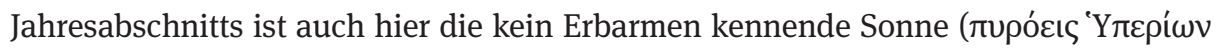
[91]); zu diesem Zeitpunkt wird die dionysische Pflanze schlechthin das Licht der Welt erblicken, die neue dionysische Ära wird eingeläutet. Mit dem Sternbild der Jungfrau sind die Ernte und der Keltervorgang der Trauben verbunden, die Voraussetzung dafür bildet der Reifungsprozess während der Hochsommertage. Wenn Nonnos durch die Setzung dieser beiden Wörter auf die Arat-Stelle verweist, so tut er dies nicht um des bloßen Zitierens willen, die sommerliche Mittagshitze dient der Einreihung der Badeszene in die chronologische Ordnung des Epos. Dionysos steht zusammen mit den sich im Wasser tummelnden und fischenden Satyrn am Vorabend der Geburt des Weines, den erst die Herbsthore und das Sternbild der Jungfrau in die Welt bringen wird, sämtliche Ereignisse um Ampelos werden von Beginn an also auch durch intertextuelle Einschaltungen vorbereitet.

56 Text: Martin (1998) 9f., Übersetzung: Erren (2009) 15.

57 Vgl. Stegemann (1930).

58 An dieser Stelle danke ich Bettina Feuchtenhofer, die mich auf die Funktion des Sternbilds Jungfrau aufmerksam machte.

59 Zur Stelle siehe S. 187-195. 
Als Folie für die einleitende Szene der Ampelos-Episode dienen neben Arat auch die kallimacheischen Hymnen. Für seine Szene des mittäglichen Bades legt Nonnos offensichtlich auch den Athene-Hymnus des Kallimachos zugrunde. Ganz ähnlich wie Dionysos nimmt die Göttin Athene ein Bad in der Mittagshitze (Call. Lav. Pall. 5,70$74):^{60}$

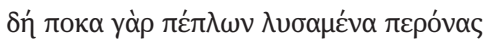

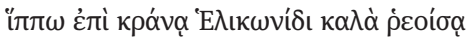

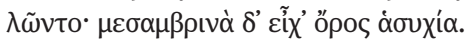

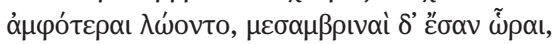

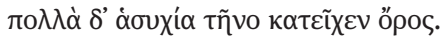

Einstmals nämlich lösten die beiden die Spangen von ihren Gewändern und badeten in der helikonischen Pferdequelle, der schönfließenden. Mittägliche Stille beherrschte den Berg. Beide badeten, es war die Mittagsstunde, tiefe Stille hatte diesen Berg ergriffen.

Nonnos scheint nicht nur kleine sprachliche und inhaltliche Details zu übernehmen

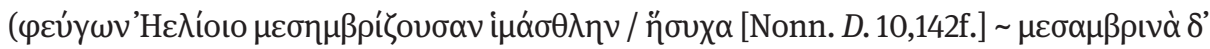

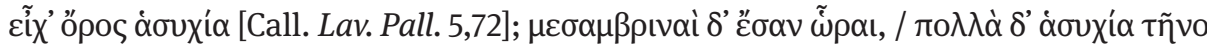

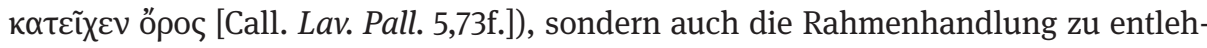
nen. Im kallimacheischen Hymnus ist nämlich vom Bad zweier Personen die Rede: Athene steigt zusammen mit ihrer Lieblingsbegleiterin Chariklo in die Fluten (Call. Lav. Pall. 5,55-69), die in den Versen 58 und 59 kurz charakterisiert und in Vers 67 namentlich genannt wird: ${ }^{61}$

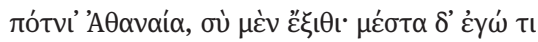

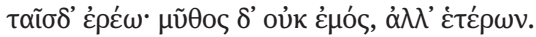

55

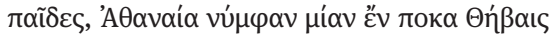

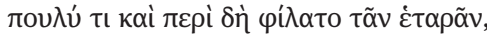

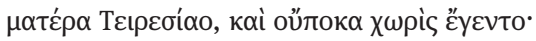

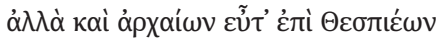

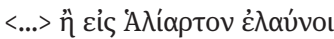



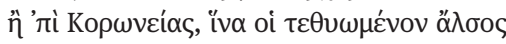

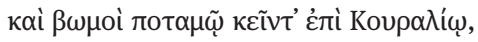

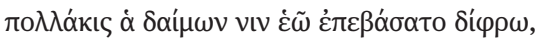

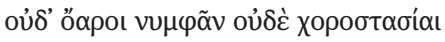

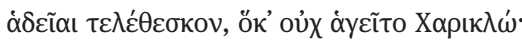

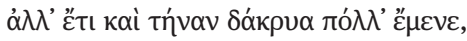

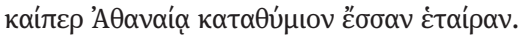

Athene, Herrin, komm doch heraus! Ich will in der Zwischenzeit diesen hier etwas erzählen; die Geschichte stammt aber nicht von mir, sondern von anderen. Mädchen, Athene liebte einst eine Nymphe in Theben sehr und weit mehr als ihre anderen Gefährtinnen, die Mutter des Teiresias.

60 Text: Pfeiffer (1953) 32, Übersetzung: Asper (2004) 442f. Zum Vergleich der beiden Stellen siehe Chrétien (1985) 138 Anm. ad 141-175.

61 Text: Pfeiffer (1953) 31f., Übersetzung: Asper (2004) 440-443. 


\begin{abstract}
Niemals trennte sie sich von ihr, (60) sondern wenn sie im ehrwürdigen Thespiae [etwa: verweilte] oder nach Haliartos die Rosse lenkte, auf der Fahrt durch das Ackerland der Boioter, oder nach Koroneia, wo ihr duftender Hain liegt und ihre Altäre am Fluss Kuralios, (65) oftmals ließ die Gottheit sie dann auf ihren eigenen Wagen steigen. Und weder die fröhlichen Lieder der Nymphen noch der Reigentanz waren ihr angenehm, wenn Chariklo sie nicht anführte. Doch auf diese sollten noch viele Tränen warten, mochte sie auch Athenes Lieblingsgefährtin sein.
\end{abstract}

Die Figurenkonstellation ist hier wie dort ähnlich. Beide, Athene bei Kallimachos und Dionysos bei Nonnos, sind in Liebe zu einem Gefährten entbrannt und zeigen die typischen Anzeichen dafür: die Freude bei der Anwesenheit des Geliebten bzw. die Trauer bei dessen Abwesenheit (vgl. Nonn. D. 10,217-277). Beide Götter nehmen gemeinsam mit ihrem Liebling ein Bad, und beiden Lieblingen ist ein schweres Schicksal beschieden: Ampelos wird durch den Sturz von einem Stier den Tod finden und Chariklo die Erblindung ihres Sohnes Teiresias beklagen, der unrechtmäßigerweise Athene beim Baden beobachtet hat. Nonnos arbeitet auch hier wieder höchst assoziativ, niemals entsprechen die Mythen einander genau, stets räumt der Dichter sich das Recht auf poetische Ungezwungenheit und Autonomie ein.

\title{
2.7 Zwischen Heiden- und Christentum - Nonnos und die Spätantike
}

Die einleitende Szene der Ampelos-Episode enthält auch Aspekte, die kulturellen Ausdrucksformen der Spätantike verpflichtet sind. Als Inspirationsquelle für die Komposition der Szene um die badenden Satyrn führt Gennaro D’Ippolito die sog. idromimi oder mimi aquatici ins Treffen, Wasserspiele, die besonders im 4. und 5. Jh. n. Chr. hohe Popularität genossen. ${ }^{62}$ Für den spätantiken griechischen Osten sind beispielsweise bei Johannes Malalas aquatische Spiele anlässlich des Maiuma-Festes belegt, Mysterien, die in Antiochia einmal jährlich im Frühling für Dionysos und Aphrodite abgehalten wurden (Jo. Mal. chron. 12,3 Thurn [= 285 Dind.]). ${ }^{63}$

Diese möglichen kulturgeschichtlichen Einflüsse werfen eine neue Frage auf: Wie können in der christlichen Welt des 5. und 6. Jhs. n. Chr. derartige Festlichkeiten Bestand haben? Denn nicht nur in Antiochia ist ein solches Dionysos-Fest belegt, sondern auch für Gaza im 6. Jh. n. Chr., wo an den zur Wintersonnenwende abgehaltenen Brumalia dem Gott des Weines und der Ernte gehuldigt wurde. ${ }^{64}$ Das Argument, dass sich gerade im gräko-syrischen Kulturraum die pagane Religion besonders lange gehalten hat, mindestens bis ins 5. Jh. n. Chr., vermag das scheinbare Paradoxon nur

62 Vgl. D’Ippolito (1962); D’Ippolito (1964) 100, 133, 145f.; Gigli Piccardi (2003) 629; Webb (2008) 100-102.

63 Vgl. Belayche (2004) 16-19. Zu den lateinischen Quellen für Wasserspiele vgl. Traversari (1960) 55-61.

64 Vgl. Jo. Mal. chron. 7,7 Thurn (=178-180 Dind.); Chor. XIII (= Dial. 7) Foerster u. Richtsteig; siehe Belayche (2004) 14. 
unvollständig zu erklären. Denn mindestens ebenso bedeutsam für den kulturellen Dialog der Zeit war der Umgang des Christentums mit den klassischen Traditionen. Pagane Elemente und Zeremonien waren Teil des neuen religiösen Selbstverständnisses, heidnische Mythologie wurde ihrer religiösen Konnotation enthoben und somit für eine literarische Verarbeitung fruchtbar gemacht. ${ }^{65}$ Die Einordnung der Dionysiaka in ein solches kulturelles Milieu fördert ganz besonders das Verständnis für diese Art von epischer Dichtung. Die Frage nach der Konfession des Autors, die am Beginn der Nonnos-Forschung im Vordergrund stand, wird obsolet, die Dionysiaka und die Paraphrase des Johannes-Evangeliums, die auf den ersten Blick so unvereinbar erscheinen, werden dadurch erst vollständig erklär- und interpretierbar. Noch in der zweiten Hälfte des 5. Jhs. n. Chr. waren die Dichter ob ihres hohen Bildungsgrades fähig, mit Texten und Motiven beider Religionen kreativ umzugehen.

Vor allem die Ampelos-Episode wurde und wird immer wieder vor christlichem Hintergrund gesehen: Das in der ersten Szene dominierende Wasser wandelt sich bis zum Ende des 12. Buches in Wein, ${ }^{66}$ und die mehrfachen Prophezeiungen über die bevorstehende Geburt der neuen Pflanze werden als Spezifikum eines christlichen Weltbildes verstanden. Nonnos verfasst mit der Paraphrase des Johannes-Evangeliums nicht nur ein rein christliches Werk, sondern scheint auch in die heidnischen Dionysiaka vermehrt Motive aufgenommen zu haben, die aus der Bibel geläufig sind. Schon Paul Collart weist auf die Parallelen zwischen der Ampelos-Episode und der biblischen Hochzeit zu Kana hin, die Nonnos in der Paraphrase in aller Ausführlichkeit schildert (Nonn. P. 2). Dionysos' Nähe zur christlichen Religion und zu Jesus Christus in seiner Funktion als Erlöser der Menschheit sowie die allgemein beliebten synkretistischen Tendenzen der Zeit steuern das Übrige zu dieser Interpretation bei. ${ }^{67}$

In dieser ersten Szene würdigt der Dichter jedoch ausschließlich Dionysos und das Kollektiv der Satyrn, Ampelos, der zusammen mit Dionysos der Protagonist der folgenden Ereignisse ist, wird noch mit keinem Wort erwähnt. Ein Grund dafür liegt in der Gesamtkonzeption der Episode. Der Schwerpunkt liegt auf dem Weingott Dionysos, Ampelos steht lediglich vorübergehend im Zentrum des Interesses, und zwar nur bis zur Geburt des Weines. Er ist als Figur bis zu dem Zeitpunkt wichtig, wo Dionysos sein Hauptattribut verliehen bekommt. Innerhalb dieses auf Dionysos ausgerichteten kompositorischen Rahmens malt der Dichter dennoch ein lebendiges Bild von dem ansonsten wenig bekannten Satyrn. Das folgende Kapitel begibt sich auf die Spur des Ampelos-Mythos. Ist dieser auch vor Nonnos greifbar? Welches Charakterbild wird in den Dionysiaka für Ampelos entworfen und woraus konstituiert es sich? Beruht die Gestaltung der Figur allein auf Nonnos' kreativem Geist und dichterischer Imagination oder stützt sich der Autor auch auf bestimmte literarische oder mythische Traditionen?

65 Vgl. Belayche (2004) 20.

66 Vgl. Collart (1930) 9; Gigli Piccardi (2003) $733 \mathrm{f}$.

$67 \mathrm{Zu}$ den motivischen Verbindungen zwischen Dionysos und Jesus vgl. etwa Noetzel (1960); zur Auseinandersetzung christlicher, jüdischer und neuplatonischer Literaten mit Dionysos siehe Baeumer (2006) 65-86. Zu christlichen Elementen in der Ampelos-Episode siehe Kap. 8.3. 